\title{
Security-Constrained Generation and Transmission Outage Scheduling With Uncertainties
}

\author{
Lei Wu, Member, IEEE, Mohammad Shahidehpour, Fellow, IEEE, and Yong Fu, Member, IEEE
}

\begin{abstract}
This study presents a stochastic model for the independent system operator's (ISO's) optimal coordinated long-term maintenance scheduling of generation units and transmission lines with short-term security-constrained unit commitment (SCUC). Random disturbances of power systems including forced outages of generation units and transmission lines, load forecast errors, and fuel price fluctuations are modeled as scenario trees using the Monte Carlo simulation. Lagrangian relaxation (LR) is applied to separate the coordinated optimization problem into long-term equipment maintenance (LTEM) and stochastic long-term SCUC (LTSCUC) subproblems. For the stochastic LTSCUC subproblem, scenario bundle constraints are relaxed via $L R$ and the optimization problem is decomposed into deterministic LTSCUC problems. LR is applied to each deterministic LTSCUC to relax long-term fuel and emission limits and decompose the problem into shortterm SCUC subproblems. The decomposition is further applied to short-term SCUC subproblems for separating hourly unit commitment and transmission network constraints. The unit commitment is formulated as a mixed-integer programming (MIP) problem and solved by the branch-and-cut method using CPLEX. The outcome of this study includes the hourly scheduling of outages of generation units and transmission lines, which corresponds to the optimal generation unit commitment and dispatch, and transmission flows. The hourly schedules minimize the total cost of operation and maintenance and satisfy long-term and short-term constraints of generation units and transmission network with the inclusion of power system uncertainty. A modified IEEE 118-bus system is used to exhibit the effectiveness of the proposed scheduling approach.
\end{abstract}

Index Terms-Optimal coordination of generation and transmission outage scheduling, security-constrained unit commitment, stochastic modeling of power systems.

\section{NOMENCLATURE}

Variables:

$F_{c, i t p}(\cdot)$

$I_{i t p}^{s}$

$J_{l t p}^{s}$

$L O L E^{s}$
Production cost function of unit $i$ at time $t$ at weekly interval $p$.

Commitment state of unit $i$ at time $t$ at weekly interval $p$ in scenario $s$.

State of line $l$ at time $t$ at weekly interval $p$ in scenario $s$.

Loss-of-load-expectation (LOLE) of scenario $s$.

$\overline{L O L E}$
$L S_{b t p}^{s}$
$P_{i t p}^{s}$
$P_{b t p}^{s}$
$q_{l \tau}, r_{l \tau}$
$S U(D)_{i t p}^{s}$
$V P$
$V L_{E M}$
$V L^{s}$
$V L P$
$V L L$

$V L R$ Manuscript received May 20, 2009; revised December 03, 2009. First pub-
lished February 18, 2010; current version published July 21, 2010. Paper no. TPWRS-00366-2009.

The authors are with the Electrical and Computer Engineering Department, Illinois Institute of Technology, Chicago, IL 60616 USA (e-mail: 1wu10@iit. edu; ms@iit.edu; fuyong@iit.edu).

Digital Object Identifier 10.1109/TPWRS.2010.2040124
$X_{i t p}$

$Y_{l t p}$

$\sigma_{L O L E}$

$\eta, \pi, \theta, \alpha, \beta$

$i, k, l, m, n, p, s, t, \tau$

Constants:

$D_{i t p}$

$e_{i}, l_{i}$

$E_{l t p}$

Expected quantity of loss-of-loadexpectation for all $S$ scenarios. Load shedding at bus $b$ at time $t$ at weekly interval $p$ in scenario $s$.

Power generation dispatch of unit $i$ at time $t$ at weekly interval $p$ in scenario $s$.

System demand at bus $b$ at time $t$ at weekly interval $p$ in scenario $s$. Indicators for beginning and end maintenance of line $l$ at time $\tau$. Startup/shutdown cost of unit $i$ at time $t$ at weekly interval $p$ in scenario $s$. Objective value of a feasible solution of the original problem.

Value of optimal LR function of the maintenance subproblem.

Value of optimal LR function of the SCUC subproblem in scenario $s$. Value of a feasible solution of the stochastic SCUC subproblem. Value of optimal LR function of the stochastic SCUC subproblem with relaxed bundle constraints.

Sum of optimal LR function values of maintenance and stochastic SCUC subproblems with relaxed bundle constraints.

Maintenance status of unit $i$ at time $t$ at interval $p, 0$ if unit is offline for maintenance, otherwise 1.

Maintenance status of line $l$ at time $t$ at interval $p, 0$ if line is offline for maintenance, otherwise 1.

Estimated standard deviation of LOLE.

Lagrangian multipliers.

Indexes.

Maintenance cost of unit $i$ at time $t$ at interval $p$.

Starting and ending time for maintenance window of equipment $i$. Maintenance cost of line $l$ at time $t$ at interval $p$.

$M D$ Maintenance duration.

$M N_{\max , l}$
Partial maintenance number upper limit of line $l$. 


$\begin{array}{ll}M T_{\min , l}^{\text {fff }} & \begin{array}{l}\text { Minimum time interval between two } \\ \text { consecutive partial maintenances of } \\ \text { line } l .\end{array} \\ M T_{\min , l}^{\text {Mn }} & \begin{array}{l}\text { Minimum duration for partial } \\ \text { maintenance of line } l .\end{array} \\ N B & \text { Number of buses. } \\ N G & \text { Number of units. } \\ N L & \text { Number of transmission lines. } \\ N P & \text { Number of weeks under study. } \\ N T & \text { Number of hours at each weekly } \\ P^{s} & \text { interval (168 h). } \\ P_{\min , i} & \text { Weight of scenario } s . \\ P_{\max , i} & \text { Minimum capacity of unit } i . \\ P_{D, t p}^{s} & \text { Maximum capacity of unit } i . \\ p v_{b t p}^{s} & \text { System demand at time } t \text { at weekly } \\ & \text { interval } p \text { in scenario } s . \\ S & \text { Value of lost load (VOLL) of bus } b \text { at } \\ \sigma_{f i x} & \text { time } t \text { at weekly interval } p \text { in scenario } \\ \varepsilon_{1}, \varepsilon_{2} & s . \\ & \text { Total number of scenarios. } \\ & \text { Predefined standard deviation of } \\ \text { LOLE. } & \text { Predefined convergence threshold. }\end{array}$

\section{INTRODUCTION}

$\mathbf{R}$ ESTRUCTURED power systems signify generation and transmission participants that are unbundled as self-interested entities which are also responsible for their maintenance outage scheduling in a competitive environment. In such environments, generation companies (GENCOs) submit their maintenance plans and constraints to the independent system operator (ISO) including maintenance time windows, available maintenance resources, and generation price offers. Transmission companies (TRANSCOs) also submit their respective maintenance plans and constraints to the ISO. The ISO is responsible for the optimal coordination of maintenance for generation units and transmission lines which would ensure the security of power systems and maximize the social welfare [1]-[3].

The study in [4]-[7] focused on generation and transmission maintenance and their coordination. However, the subject of transmission security in restructured power systems was not considered. Furthermore, random outages of power systems were not included when maximizing planned outages in long-term operation planning studies.

Our proposed coordinated long-term generation and transmission maintenance scheduling intends to coordinate generation and transmission outage scheduling with short-term security-constrained generation scheduling. Random outages of generators and transmission lines, load forecast errors, and fuel price fluctuations are considered which could influence the optimal maintenance outage scheduling. Such a detailed security-constrained maintenance scheduling approach provides a wider range of options for managing short-term economics. Mathematically, the proposed problem is a stochastic, large-scale mixed-integer optimization problem and we introduce an effective decomposition and coordination strategy for its solution. The Lagrangian relaxation (LR) technique is applied to decompose such a stochastic and large mixed-integer programming (MIP) problem into tractable small-scale subproblems.

The proposed stochastic model could be applied to a vertically integrated utility. Here we consider fuel and emission constraints when scheduling the long-term operation of a utility. The same model can be utilized by centralized energy markets with ISOs. Here a combination of fuel and emission constraints used by traditional utilities, which is a quadratic function of power generation, represents the energy constraint for a GENCO. That is, GENCOs submit energy limits which indicate that the sum of daily generation for individual units, or the entire GENCO, would not exceed a prescribed level. The energy constraint is utilized by security-constrained unit commitment (SCUC) which is a market clearing tool in the day-ahead energy market.

The attributes of the proposed stochastic model, for the coordination of long-term generation and transmission maintenance schedules with the short-term SCUC, are presented in the following:

1) Coordination of generation maintenance with transmission maintenance. We introduce a binary variable for representing the transmission line status and adopt an optimization technique that applies to both generation and transmission maintenance scheduling.

2) LR is used to decompose the original stochastic problem into long-term equipment maintenance (LTEM) and long-term SCUC (LTSCUC) with fuel and emission subproblems, with unit commitment and transmission line status as coordinating (coupling) variables. The LTEM and LTSCUC subproblems are solved individually and violations are identified. Lagrangian multipliers are updated iteratively for subproblems until the stopping criterion is met.

3) Random outages of generators and transmission lines, load forecast errors, and fuel price fluctuations are simulated by the Monte Carlo method when solving the LTSCUC subproblem. The uncertainty could influence the maintenance schedule when updating Lagrangian multipliers. The scenario-based technique is used to solve the stochastic LTSCUC subproblem. Scenario reduction is adopted when solving the stochastic LTSCUC as a tradeoff between computation time and solution accuracy. The LR method is adapted to relax bundle constraints and a modified surrogate subgradient method is used for a faster convergence.

4) The chronological load profile is considered in the LTEM scheduling and its coordination with the short-term SCUC.

The rest of the paper is organized as follows. Section II describes the proposed model and its solution methodology. Section III presents the detailed formulation of maintenance and generation scheduling subproblems. The coordination and solutions methodologies are presented in Section IV. Section V presents and discusses a modified IEEE 118-bus system with 54 units and 91 loads. The conclusion is drawn in Section VI. 


\section{StOchastic MODEL DescriPtion}

In this paper, we analyze the optimal maintenance scheduling of a vertically integrated utility. The same methodology would also apply to restructured power systems. The utility's objective is to minimize the hourly cost of security-constrained short-term operation and long-term maintenance.

In applying (1) to a vertically integrated utility, the first two terms represent the cost of maintenance for generation and transmission equipment respectively. The remaining terms represent the production cost. We could consider a variable hourly maintenance cost coefficients $\left(D_{i t p}\right.$ and $\left.E_{l t p}\right)$ for each equipment. If the proposed model is used in restructured power systems, GENCOs and TRANSCOs would be independently responsible for generation and transmission maintenance, no equipment maintenance cost will appear in (1), and $D_{i t p}$ and $E_{l t p}$ would be zero. The ISO would use the objective (1) to schedule equipment maintenance outages based on a GENCO's or a TRANSCO's preferred maintenance schedule. Accordingly, $D_{i t p}$ and $E_{l t p}$ will be zero during the most preferred periods for maintenance of the corresponding equipment in order to increase the chance of maintenance during such periods:

$$
\begin{aligned}
\operatorname{Min}\left\{\sum_{p=1}^{N P} \sum_{t=1}^{N T} \sum_{i=1}^{N G} D_{i t p} \cdot\left(1-X_{i t p}\right)\right. \\
\quad+\sum_{p=1}^{N P} \sum_{t=1}^{N T} \sum_{l=1}^{N L} E_{l t p} \cdot\left(1-Y_{l t p}\right)+\sum_{s=1}^{S} P^{s} \\
\\
\quad \sum_{p=1}^{N P} \sum_{t=1}^{N T}\left\{\sum_{i=1}^{N G}\left[F_{c, i t p}\left(P_{i t p}^{s} \cdot I_{i t p}^{s}\right)+S U_{i t p}^{s}+S D_{i t p}^{s}\right]\right. \\
\left.\left.+\sum_{b=1}^{N B} p v_{b t p}^{s} \cdot L S_{b t p}^{s}\right\}\right\}
\end{aligned}
$$

Such objectives may not be easily realized by a one-step optimization technique due to the enormity of the maintenance scheduling problem. Thus, we schedule the long-term equipment maintenance and the short-term unit commitment problems separately, which are based on the decomposition technique depicted in Figs. 1-3. The distinct features of the proposed model include the way we introduce stochastic variables in the coordinated generation and transmission maintenance problem, handle scenario bundle constraints, and link maintenance and operation schedules. Fig. 1 shows the decomposition strategy of LTEM represented by (2)-(9) and LTSCUC constraints. Coupling constraints (11), (12) of LTEM and LTSCUC are relaxed using the LR method. LTEM and LTSCUC subproblems are solved individually before the coupling constraints are checked.

If the coupling constraints (11), (12) are not satisfied, Lagrangian multipliers $\eta$ and $\pi$ corresponding to the two constraints are updated and the pseudo price signals are fed back to the subproblems. We introduce $I$ as the unit commitment status variable and $J$ as the transmission line status variable, so that the transmission line maintenance can be treated the same way as that of generation units. The network security is checked in the deterministic LTSCUC subproblem and the same strategy is used for updating the Lagrangian multiplier $\eta$ and $\pi$.
Optimizing Stochastic LTEM \& LTSCUC by Solving Its Dual Problem

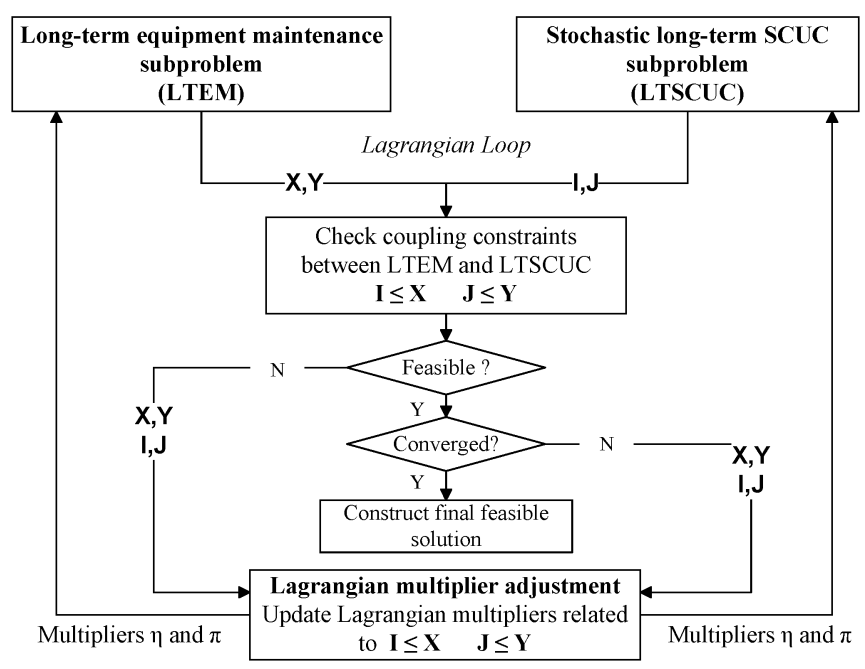

Fig. 1. Decomposition and coordination of LTEM and LTSCUC.

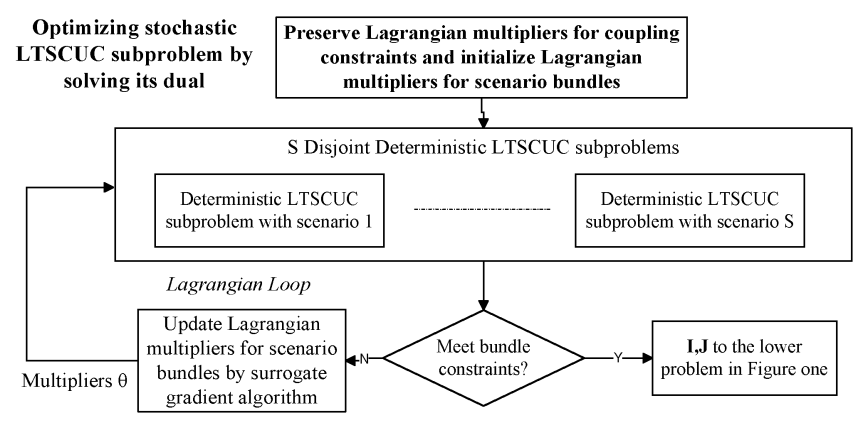

Fig. 2. Decomposition of scenario bundle constraints.

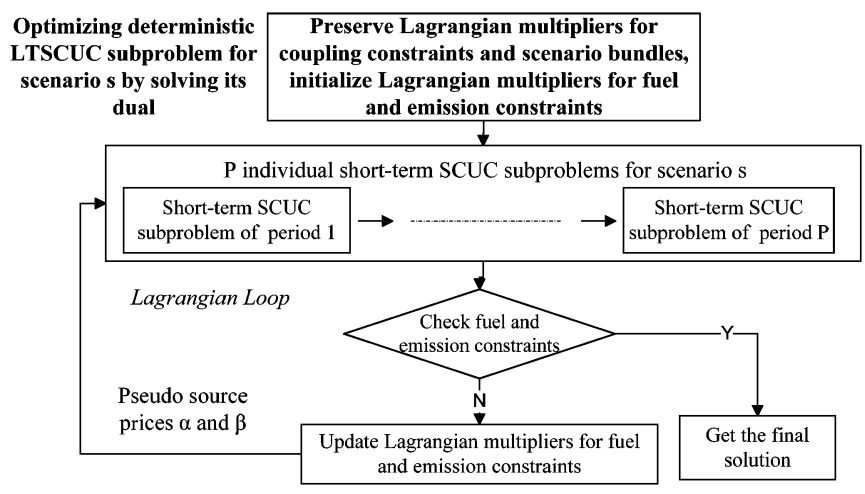

Fig. 3. Decomposition and coordination of deterministic LTSCUC.

Fig. 2 shows the decomposition strategy of the stochastic LTSCUC for managing the scenario bundle constraints (10). Scenario bundle constraints are relaxed by the LR method and the deterministic LTSCUC problem (shown in Fig. 3) is solved in each scenario. If the LTSCUC results in Fig. 2 do not satisfy the scenario bundle constraints, Lagrangian multipliers $\theta$ are updated and the corresponding pseudo price signals are fed back to scenarios for the next iterative solution. Since the problem is not convex, there is no guarantee that the bundle constraints would be entirely satisfied. Thus, the scenarios consider approximate solutions for fast convergence while maintaining a rel- 
atively high level of accuracy. The iterative process will stop when the total violation of weighted bundle constraints is below a certain tolerance. A heuristic process is then used to obtain a feasible solution for satisfying the scenario bundle constraints. We use the modified surrogate subgradient method to improve the convergence. The solution details shown in the next section illustrate a tradeoff between the computation speed and the accuracy of solution.

Fig. 3 illustrates the decomposition of the deterministic LTSCUC into tractable subproblems. Fuel and emission constraints are relaxed with resource penalty prices represented by Lagrangian multipliers $\alpha$ and $\beta$. Based on the decomposition process, the individual short-term unit commitment problems will be solved without the fuel and emission constraints.

If the results do not satisfy the fuel and emission constraints, the Lagrangian multipliers $\alpha$ and $\beta$ are updated and the corresponding penalty resource prices are fed back to solve the short-term subproblems again [8], [9].

\section{Formulation OF SUbPROBLEMS}

The LTEM and LTSCUC formulations, constraints, and coordination are summarized as follows.

1) Generation Maintenance Constraints: Generation maintenance windows: In the following, (2) shows starting and ending times when the maintenance is allowed. Also, (3) represents the total maintenance duration, and (4) shows that each unit could only be maintained once within the maintenance window:

$$
\begin{aligned}
& X_{i \tau}=0 \text { or } 1 \text { when } e_{i} \leq \tau \leq l_{i} ; \\
& \text { otherwise } X_{i \tau}=1 \forall i, \tau<e_{i} \text { or } \tau>l_{i} \\
& \sum_{\tau=e_{i}}^{l_{i}}\left(1-X_{i \tau}\right)=M D_{i} \quad \forall i \\
& \sum_{\tau=k}^{k+M D_{i}-1}\left(1-X_{i \tau}\right) \geq M D_{i} \cdot\left[X_{i(\tau-1)}-X_{i \tau}\right] \\
& \forall i, k=e_{i}, \cdots, l_{i}-M D_{i}+1 \\
& \sum_{\tau=k}^{l_{i}}\left\{1-X_{i \tau}-\left[X_{i(k-1)}-X_{i k}\right]\right\} \geq 0 \\
& \forall i, k=l_{i}-M D_{i}+2, \cdots, l_{i} .
\end{aligned}
$$

Generation maintenance resources and crew availability: The crew availability would limit the number of units that can be maintained simultaneously. For example, if units 1,2 and 3 are to be maintained simultaneously, the constraint is represented as $X_{1 \tau}=X_{2 \tau}=X_{3 \tau}$. Otherwise, for a single equipment, $\left(1-X_{1 \tau}\right)+\left(1-X_{3 \tau}\right)+\left(1-X_{3 \tau}\right) \leq 1$. If unit 1 is maintained before unit $2, \sum_{\tau=1}^{t}\left(1-X_{1 \tau}\right)-\left(1-X_{2 t}\right) \geq 0$. While $X_{1 t}=X_{2 t}$ indicates that unit 1 and 2 are to be on maintenance outage simultaneously. We can also represent limited resources for maintenance and crew availability at each location as $\sum_{i \in A} f_{R i} \cdot\left(1-X_{i \tau}\right) \leq H_{R \tau}$ where $f_{R i}$ is the resource needed for the maintenance of unit $i$ and $H_{R \tau}$ is the total resource quantity available at time $\tau$ for area A.
2) Transmission Maintenance Constraints: Partial maintenance is allowed for transmission lines. Hence

$$
\begin{aligned}
& Y_{l \tau}=0 \text { or } 1 \text { when } e_{l} \leq \tau \leq l_{l} ; \\
& \text { otherwise } Y_{l \tau}=1 \forall l, \tau<e_{l} \text { or } \tau>l_{l} \\
& \sum_{\tau=e_{l}}^{l_{l}}\left(1-Y_{l \tau}\right)=M D_{l} \forall l \\
& q_{l \tau}-r_{l \tau}=Y_{l(\tau-1)}-Y_{l \tau}, \quad q_{l \tau}+r_{l \tau} \leq 1, \\
& 1 \leq \sum_{\tau=1}^{N T \cdot N P} r_{l \tau} \leq M N_{\max , l} \forall l, \forall \tau \\
& k+M T_{\min , l}^{o n}-1 \\
& \quad \sum_{\tau=k}^{\forall}\left(1-Y_{l \tau}\right) \geq\left(Y_{l(\tau-1)}-Y_{l \tau}\right) \cdot M T_{\min , l}^{o n} \\
& \forall l, k=e_{l}, \cdots, l_{l}-M T_{\min , l}^{o n}+1 \\
& \sum_{\tau=k}^{l_{l}}\left\{1-Y_{l \tau}-\left[Y_{l(k-1)}-Y_{l k}\right]\right\} \geq 0 \\
& \forall l, k=l_{l}-M T_{\min , l}^{o n}+2, \cdots, l_{l} \\
& k+M T_{\min , l}^{o f f}-1 \\
& \quad \sum_{\tau=k} Y_{l \tau} \geq\left(Y_{l \tau}-Y_{l(\tau-1)}\right) \cdot M T_{\min , l}^{o f f} \\
& \forall l, k=e_{l}, \cdots, l_{l}-M T_{\min , l}^{o f f}+1 \\
& \sum_{\tau=k}^{l_{l}}\left\{Y_{l \tau}-\left[Y_{l k}-Y_{l(k-1)}\right]\right\} \geq 0 \\
& \forall l, k=l_{l}-M T_{\min , l}^{o f f}+2, \cdots, l_{l} .
\end{aligned}
$$

3) Stochastic LTSCUC Constraints: Short-term SCUC constraints presented in [18] are considered here in addition to

- Reliability constraints: An explicit reserve requirement constraint is not introduced. The optimal reserve requirement is implicitly determined because we add a penalty load shedding item to the objective function and include a LOLE constraint.

- Scenario bundle constraints: Scenario bundle constraints indicate that if two scenarios $\mathrm{s}$ and s' are indistinguishable from the beginning to time $(P, \tau)$ on the basis of information available at time $(P, \tau)$, then the generator commitment decisions rendered for the two scenarios must be identical from the beginning to time $\tau . B(s, P, \tau)$ represents a part of scenario $\mathrm{s}$ from the beginning to time $(P, \tau)$. In the scenario bundle constraints (10), the generator commitment decision $I$ rather than the line status $J$ is included. The line status $J$ is used as a state variable to relate the transmission maintenance decision and SCUC in (12). Based on (12), if a line is on maintenance, the line status $J$ for all scenarios would be zero; otherwise, $J$ would be one. The status of $J$ is determined by the optimization of LTSCUC because the line would be utilized in minimizing the operation cost. That is, it would not be necessary to include the line status in the scenario bundle constraints (10):

$I_{i t p}^{s}=I_{i t p}^{s^{\prime}}$, for $(p, t) \in\left\{(P, \tau) \mid B(s, P, \tau)=B\left(s^{\prime}, P, \tau\right)\right\}$. 
The detailed formulation of LTSCUC constraints can be found in our previous works [20], [22].

4) Coupling Constraints for LTEM and LTSCUC in Fig. 1: Coupling constraints of generator maintenance $X$ and unit commitment status $I(11)$ :

$$
I_{i t p}^{s} \leq X_{i t p} \quad \forall s, \forall i, \forall t, \forall p .
$$

Coupling constraints of transmission line maintenance $Y$ and transmission line status $J(12)$ :

$$
J_{l t p}^{s} \leq Y_{l t p} \quad \forall s, \forall l, \forall t, \forall p .
$$

The proposed stochastic model differs from the deterministic one in several respects. First, the objective of the proposed stochastic model is to minimize the expected production cost of all scenarios, whereas the deterministic model would only minimize the production cost of a certain deterministic case. Second, the impact of the variance of uncertain variable on the maintenance schedule is considered. We also incorporate the coupling constraints of generator maintenance and unit commitment status (11) and the coupling constraints of transmission line maintenance and transmission lines status (12). Furthermore, there are scenario bundle constraints in the stochastic LTSCUC subproblem. Hence, the solution of stochastic model which includes a set of weighted scenarios is not simply the same as solving a deterministic model separately for each scenario.

\section{SOlution OF SubProblems}

\section{A. Coupling Constraints for LTEM and LTSCUC Subproblems}

LR is employed to solve the coupling constraints (11), (12). Once constraints are relaxed and introduced into the LTEM and LTSCUC subproblems via the Lagrangian multipliers $\boldsymbol{\eta}$ and $\boldsymbol{\pi}$, the modified LTEM and LTSCUC subproblems at iteration $n$ are presented in (13) and (14), respectively:

$$
\begin{aligned}
V L_{E M}\left(\eta^{n}, \pi^{n}\right) & \\
= & \operatorname{Min} \sum_{p=1}^{N P} \sum_{t=1}^{N T} \sum_{i=1}^{N G}\left[D_{i t p} \cdot\left(1-X_{i t p}\right)-\left(\sum_{s} \eta_{i t p}^{s, n}\right) \cdot X_{i t p}\right] \\
& +\sum_{p=1}^{N P} \sum_{t=1}^{N T} \sum_{l=1}^{N L}\left[E_{i t p} \cdot\left(1-Y_{l t p}\right)-\left(\sum_{s} \pi_{l t p}^{s, n}\right) \cdot Y_{l t p}\right]
\end{aligned}
$$

S.t. constraints (2)-(9)

$$
\begin{array}{r}
\operatorname{Min} \sum_{s=1}^{S} \sum_{p=1}^{N P} \sum_{t=1}^{N T}\left\{P ^ { s } \cdot \left\{\sum _ { i = 1 } ^ { N G } \left[F_{c, i t p}\left(P_{i t p}^{s} \cdot I_{i t p}^{s}\right)\right.\right.\right. \\
\left.+S U_{i t p}^{s}+S D_{i t p}^{s}\right] \\
\left.+\sum_{b=1}^{N B} p v_{b, t p}^{s} \cdot L S_{b, t p}^{s}\right\} \\
\left.+\sum_{i=1}^{N G} \eta_{i t p}^{s, n} \cdot I_{i t p}^{s}+\sum_{l=1}^{N L} \pi_{l t p}^{s, n} \cdot J_{l t p}^{s}\right\}
\end{array}
$$

S.t. stochastic LTSCUC constraints described in Section III.

After obtaining a solution for generator maintenance $\mathbf{X}$, transmission line maintenance $\mathbf{Y}$, as well as unit commitment $\mathbf{I}$ and transmission line status $\mathbf{J}$, we check the constraints and update the corresponding multipliers $\boldsymbol{\eta}$ and $\boldsymbol{\pi}$ by applying the subgradient method [19]. The Lagrangian iteration continues until a converged solution which would meet the stopping criterion is obtained:

$$
\begin{aligned}
& \text { If } \hat{I}_{i t p}^{s, n} \geq \hat{X}_{i t p}^{n} \text {, } \\
& \eta_{i t p}^{s, n+1}=\eta_{i t p}^{s, n}+S_{G}^{u p, n} \cdot\left(\hat{I}_{i t p}^{s, n}-\hat{X}_{i t p}^{n}\right) /\left\|\hat{I}_{i t p}^{s, n}-\hat{X}_{i t p}^{n}\right\|
\end{aligned}
$$

Otherwise

$$
\eta_{i t p}^{s, n+1}=\eta_{i t p}^{s, n}+S_{G}^{\text {down }, n} \cdot\left(\hat{I}_{i t p}^{s, n}-\hat{X}_{i t p}^{n}\right) /\left\|\hat{I}_{i t p}^{s, n}-\hat{X}_{i t p}^{n}\right\|
$$

If $\hat{J}_{l t p}^{s, n} \geq \hat{Y}_{l t p}^{n}$,

$$
\pi_{l t p}^{s, n+1}=\pi_{l t p}^{s, n}+S_{L}^{u p, n} \cdot\left(\hat{J}_{l t p}^{s, n}-\hat{Y}_{l t p}^{n}\right) /\left\|\hat{J}_{l t p}^{s, n}-\hat{Y}_{l t p}^{n}\right\|
$$

Otherwise

$$
\pi_{l t p}^{s, n+1}=\pi_{l t p}^{s, n}+S_{L}^{d o w n, n} \cdot\left(\hat{J}_{l t p}^{s, n}-\hat{Y}_{l t p}^{n}\right) /\left\|\hat{J}_{l t p}^{s, n}-\hat{Y}_{l t p}^{n}\right\|
$$

where $\|\cdot\|$ stands for a Euclidean norm, $S_{G}^{u p, n}, S_{G}^{\text {down }, n}, S_{L}^{u p, n}$ and $S_{L}^{\text {down }, n}$ are step sizes at iteration $n$ for updating multipliers of constraints (11), (12). Usually a constant step size or the one that decreases with iterations such as $(1+C) /(n+C)$ or $1 /(C 1+C 2 \cdot \sqrt{n})$ would be used, where $C, C 1$, and $C 2$ are pre-defined positive constants. In [23] the bundle method was also proposed to update Lagrangian multipliers in order to improve the updating process.

Concerning the coupling constraints (11), (12) for the LTEM and LTSCUC subproblems, the original problem is non-convex with integer variables. So additional heuristics strategies are applied to derive a feasible solution when a predefined number of Lagrangian iterations or the maximum execution time is reached. In this case, the LTSCUC solution would offer a feasible solution when generation and transmission maintenance schedules are assumed fixed according to their values in the current Lagrangian solution.

\section{B. Stochastic Simulation}

We model forced outages of generation units and transmission lines as independent Markov processes, load forecast errors as uniform random variables, and fuel price fluctuations as Ornstein-Uhlenbeck processes. For an ISO model, we could replace the fuel price with bidding prices offered by market participants since the ISO does not deal with fuel prices. The solution process will still be the same as that discussed in this section. We introduce a set of scenarios in the Monte Carlo method for modeling the stochastic conditions. For stochastic programming, we assign a weight $P^{s}$ to each scenario that reflects the possibility of its occurrence.

A two-state continuous-time Markov chain model is used to represent available and unavailable states of generators and transmission lines. The parameters used for the Monte Carlo simulation are failure and repair rates of each power system component. Using failure and repair rates, forced outages are simulated for a specified time period with the assumption that the power system is at the base case at the beginning of the 
period. Power system components are assumed independent in our scenario-based simulation and the simultaneous effects of outages are presented in scenario trees. For the simulation of load forecast errors, we divide the entire scheduling horizon into several time intervals. For each time interval we create additional scenarios based on historical data. The probabilities are assigned to hourly load quantities according to the likelihood of their occurrence. The proposed scenarios in each time interval reflect the representative days/hours chosen for each week/season.

The proposed approach makes it easy for a maintenance scheduler to include the relevant data of a certain week/season after assigning a suitable weight to each load scenario. It is desirable that one could shift the maintenance schedule to periods with higher fuel prices for mitigating higher operation cost associated with maintenance outages. We employ the Ornstein-Uhlenbeck process to simulate random fluctuations in fuel price [20], [21]. According to Fig. 4, we generate a set of scenarios, each representing possible fluctuations in fuel price, load forecast errors, as well as the availability of generation units and transmission lines.

In the proposed model, we use a low-discrepancy Monte Carlo method, Latin hypercube, to generate a set of scenarios. Weights for all scenarios in the initial scenario catalog generated by the Monte Carlo method are the same. Scenario reduction is adapted for a tradeoff between solution accuracy and computation speed, because computation requirements for solving scenario-based optimization models would depend on the number of scenarios [13], [14]. Here we use the following criterion for the stochastic LTSCUC subproblem while taking into account the solution accuracy:

$$
\begin{aligned}
& \sigma_{L O L E}=\sqrt{\sum_{s=1}^{S} P^{s} \cdot\left(L O L E^{s}-\overline{L O L E}\right)^{2} / S \cdot(S-1)} \\
& \sigma_{L O L E}<\sigma_{f i x} \quad \overline{L O L E}=\sum_{s=1}^{S} P^{s} \cdot L O L E^{s}
\end{aligned}
$$

The results are assumed to be reasonably accurate when the estimated standard deviation of LOLE, $\sigma_{L O L E}$, is less than the predefined boundary $\sigma_{f i x}$.

\section{Scenario Bundle Constraints}

The sample scenarios for representing the uncertainty are solved and a good combination of solution outcomes is selected to represent the stochastic solution. The solution outcome would satisfy the constraint that if two scenarios are indistinguishable from the beginning to time $\tau$ on the basis of information available at time $\tau$, then the decision rendered for two scenarios must be identical from the beginning to time $\tau$.

The LR method is adopted to relax the scenario bundle constraints. Thus the stochastic problem is divided into several tractable subproblems for each scenario. Once the scenario

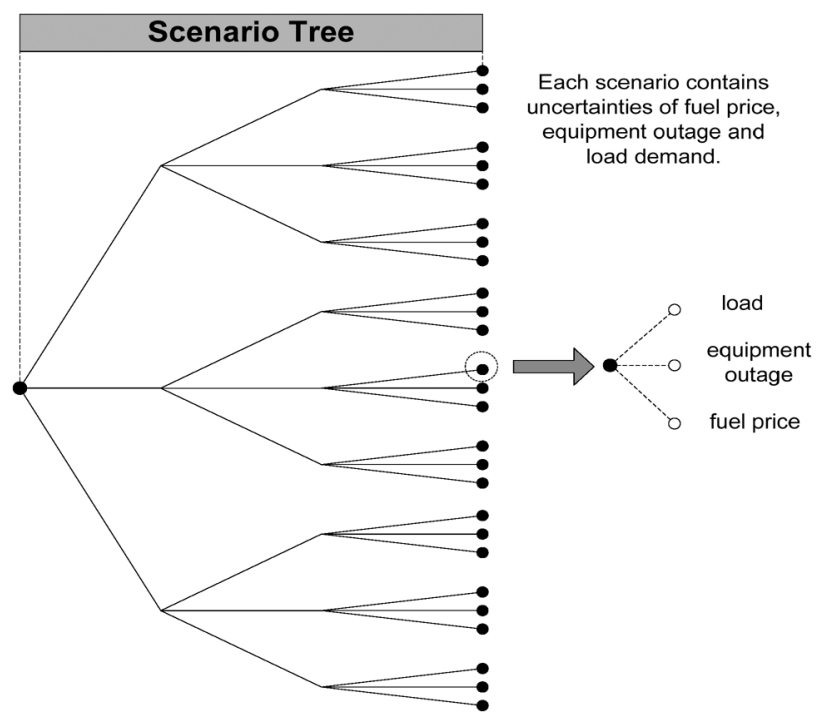

Fig. 4. Representation of uncertainty in a scenario tree.

bundle constraints are relaxed, the LTSCUC subproblem for each scenario is represented as

$$
\begin{gathered}
V L^{s}\left(\theta^{k}\right)=\operatorname{Min} \sum_{p=1}^{N P} \sum_{t=1}^{N T}\left\{P ^ { s } \cdot \left\{\begin{array}{c}
\sum_{i=1}^{N G}\left[F_{c, i t p}\left(P_{i t p}^{s} \cdot I_{i t p}^{s}\right)\right. \\
\left.+S U_{i t p}^{s}+S D_{i t p}^{s}\right]
\end{array}\right.\right. \\
\left.+\sum_{b=1}^{N B} p v_{b, t p}^{s} \cdot L S_{b, t p}^{s}\right\} \\
\left.+\sum_{i=1}^{N G} \eta_{i t p}^{s, n} \cdot I_{i t p}^{s}+\sum_{l=1}^{N L} \pi_{l t p}^{s, n} \cdot J_{l t p}^{s}\right\} \\
+\sum_{p=1}^{N P} \sum_{t=1}^{N T} \sum_{s \in B(s, P, \tau)=\Omega_{P \tau}} \theta_{i t p}^{s, k} \cdot I_{i t p}^{s}
\end{gathered}
$$

S.t. stochastic LTSCUC constraints described in Section III except scenario bundle constraints.

The target $c_{i t p}$, which is the weighted average of decision, is the same for all scenarios. Let $\theta_{i t p}^{s, k}$ be the $k$ th iteration of Lagrangian multiplier corresponding to $I_{i t p}^{s}=c_{i t p}$, $i=1 \cdots N G,(p, t) \in\{P, \tau\}$. We obtain a new decision by calculating the expected value of $I_{i t p}^{s, k}$ as $c_{i t p}^{k}$, updating $\theta_{i t p}^{s, k+1}$, and continuing the iteration. The iteration is terminated when the total weighted violation of bundle constraints, $\sum_{p=1}^{N P} \sum_{t=1}^{N T} \sum_{s \in B(s, P, \tau)=\Omega_{P \tau} P^{s}} \cdot\left|I_{i t p}^{s}-c_{i t p}\right|$, is below a certain threshold. Here, $|\cdot|$ denotes the absolute value. In order to accelerate the convergence, the surrogate gradient algorithm is adopted. The process starts by finding a scenario $s$ which has the largest weighted violation $\sum_{p=1}^{N P} \sum_{t=1}^{N T} P^{s} \cdot\left|I_{i t p}^{s}-c_{i t p}\right|$, updating multipliers, and calculating the scenario $s$ only. The detailed steps are given in Appendix A. The optimality conditions for the proposed dual solution via the surrogate subgradient method was provided in [15], [16]. Here, multipliers move iteratively closer to their global optimal.

In each scenario, the LR algorithm is adopted for the solution of deterministic long-term SCUC problem which deals 
with fuel and emission constraints. The LR will divide the original problem into several tractable subproblems, i.e., short-term SCUC subproblems based on MIP. The Lagrangian multipliers are penalty price signals which optimize the allocation of fuel consumption and emission allowance based on a hybrid subgradient and Dantzig-Wolfe method [8], [17], [18].

The iteration will be terminated when the total weighted violation of bundle constraints is below a certain threshold. Accordingly, a heuristic process will be used to introduce a feasible solution with respect to the scenario bundle constraints. Here, we find the largest weighted violation $P^{s} \cdot\left|I_{i t p}^{s}-c_{i t p}\right|$ and adjust the multiplier $\theta_{i t p}^{s}$ corresponding to hour $t$ at period $p$ of scenario $s$. The subproblem for the period $p$ of scenario $s$ is solved again and new $c_{i t p}$ are computed. The process will be repeated for finding the next maximum violation and terminated within a finite number of iterations.

There are two main LR loops. $V L_{E M}\left(\eta^{n}, \pi^{n}\right)$ in (13) and $V L^{s}\left(\theta^{k}\right)$ in (16) are the optimal LR function values for the LTEM and LTSCUC subproblems in scenario $s$ at the current iteration. The optimal LR function of the stochastic LTSCUC subproblem with the relaxed bundle constraints $V L L$ at the current iteration is calculated by (17). The sum of optimal LR function values for the maintenance and the stochastic LTSCUC subproblems with relaxed bundle constraints $V L R$ at the current iteration is calculated by (18). The Lagrangian iterations will continue until the relative difference (duality gap) between $V P$ (upper bound) and $V L R$ (lower bound) of the optimal solution is within a pre-defined tolerance as stated in (19):

$$
\begin{aligned}
& V L L=\sum_{s} V L^{s}\left(\theta^{k}\right) \\
& V L R=V L_{E M}\left(\eta^{n}, \pi^{n}\right)+\sum_{s} V L^{s}\left(\theta^{k}\right) \\
& |(V P-V L R) / V L R| \leq \varepsilon_{1} .
\end{aligned}
$$

Appendix B provides a proof for the validation of stopping criterion. The maximum number of iterations would have to be set since the duality gap could be intrinsic to the non-convex problem and would not be further reduced.

\section{CAse Studies}

A modified IEEE 118-bus system is considered in this section to demonstrate the proposed approach. The intention is to schedule the hourly maintenance, unit commitment, and generation economical dispatch over a 672-h (i.e., four weeks) horizon. The 118-bus system is composed of 54 thermal units, 186 branches, and 91 loads. The test data for the 118-bus system are given in http://motor.ece.iit.edu/data/coop. The annual peak load of the system is $6000 \mathrm{MW}$ and the load trend over the study horizon is shown in Fig. 5.

For this system, three generators are considered for maintenance, each can be on maintenance at any hour. Only one transmission line L51 is considered for maintenance and a partial maintenance is acceptable. The maximum allowable number of partial maintenances for this line is two. The partial maintenance needs to last at least eight hours with a minimum of eight hours between two consecutive partial maintenances. The concern of

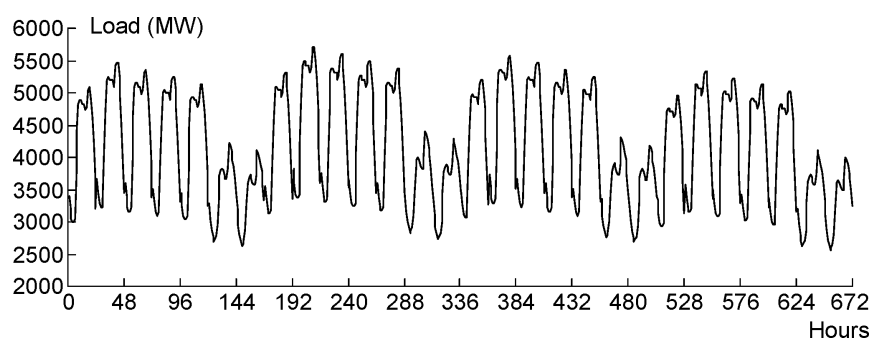

Fig. 5. Load profile in a 672-h horizon for the 118-bus system.

TABLE I

GENERATOR MAINTENANCE Limits FOR CASES 1-3

\begin{tabular}{l|c|c|c|c}
\hline Unit & At Bus & Windows & $\begin{array}{c}\text { Duration } \\
\text { (hours) }\end{array}$ & $\begin{array}{c}\text { Cost } \\
\text { (\$/hour) }\end{array}$ \\
\hline $\mathrm{U} 10$ & 25 & $1-624$ & 24 & 1200 \\
\hline $\mathrm{U} 20$ & 49 & $1-624$ & 24 & 1000 \\
\hline $\mathrm{U} 34$ & 76 & $1-624$ & 24 & 400 \\
\hline
\end{tabular}

TABLE II

TRANSMISSION LINE MAINTENANCE Limits FOR CASES 1-3

\begin{tabular}{c|c|c|c|c|c}
\hline Line & From Bus & To Bus & Windows & $\begin{array}{c}\text { Duration } \\
\text { (hours) }\end{array}$ & $\begin{array}{c}\text { Cost } \\
\text { (\$/hour) }\end{array}$ \\
\hline L51 & 38 & 37 & $1-336$ & 18 & 5000 \\
\hline $\begin{array}{c}\text { Maximum number } \\
\text { of partial } \\
\text { maintenances }\end{array}$ & \multicolumn{2}{|c|}{$\begin{array}{c}\text { Minimum duration of } \\
\text { partial maintenance } \\
\text { (hours) }\end{array}$} & $\begin{array}{c}\text { Minimum interval } \\
\text { between partial } \\
\text { maintenances (hours) }\end{array}$ \\
\hline \multicolumn{2}{c|}{2} & \multicolumn{2}{c|}{8} \\
\hline
\end{tabular}

this paper is to study the impact of uncertainties, on the coordinated maintenance scheduling of generation units and transmission lines with SCUC. Thus in the case study, emission limits and fuel consumption constraints are relaxed by introducing very large emission and fuel upper limits. The application of LR for relaxing emission and fuel consumption constraints, procedures for obtaining feasible solutions, and the performance of the system was discussed in [9] and [20].

We study four maintenance cases for a 672 -h (four-week) period:

Case 1) Uncoordinated maintenance and unit commitment schedules.

Case 2) Coordinated generation unit and transmission line maintenance schedule without the consideration of uncertainty. We refer to this case as the expected value problem since expected values are used for load forecast and fuel price.

Case 3) Impact of uncertainty on the coordinated schedule.

Case 4) Same as Case 3 with a three-day maintenance duration.

For the first three cases, the equipment maintenance data listed in Tables I and II are used. In Case 4, the same data as those of Cases 1-3 are used except the maintenance duration is increased to $72 \mathrm{~h}$. These cases are discussed as follows.

Case 1: LTEM and LTSCUC subproblems are not coordinated, so the optimal maintenance schedules are obtained individually for generators and transmission line maintenance subproblems as described in part one and two of Section III. The optimal maintenance schedule is shown in Fig. 6. 


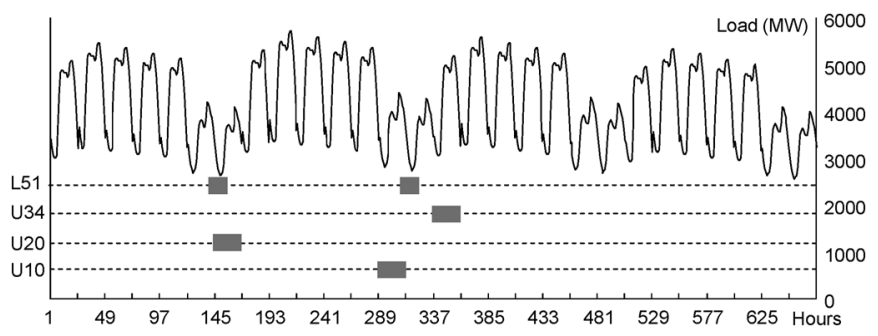

Fig. 6. Equipment maintenance in Case 1.

TABLE III

ClassificATION OF UnITS

\begin{tabular}{c|c|c}
\hline Class & Units \\
\hline Base & 451011202124252728293639404344 & 45 \\
\hline Intermediate & 12361213313238425054 \\
\hline Peaking & $78914151617181922 \quad 3 \quad 2634$ \\
& 35374748515253 & \\
\hline Off & 3033414649 \\
\hline
\end{tabular}

The maintenance of units U10, U20, and U34 are scheduled at hours of 289-312, 145-168, and 337-360, respectively. The maintenance of line L51 is scheduled at hours 144-152 and $312-320$. Based on the above maintenance schedule, we optimize the hourly unit commitment schedule for $672 \mathrm{~h}$ without the consideration of system uncertainty. The total cost is $\$ 42133556$, which includes the generator maintenance cost of $\$ 62400$, line maintenance cost of $\$ 90000$, and operation cost of $\$ 41981$ 156. In the uncoordinated case, the unit commitment schedule is not considered in the maintenance problem. If the proposed maintenance schedule is used as input to the unit commitment problem, the commitment may be more expensive or could result in load shedding. For example in Fig. 6, L51 and U20 are both maintained during hours 145-152. An inefficient utilization of generators and lines at this period could increase the total cost and/or result in load shedding.

Case 2: This case coordinates the equipment maintenance outage and the unit commitment schedule without considering any uncertainty. Table III shows, based on the percentage of time, the units that are committed as base (committed more than $85 \%$ of the time in four weeks), intermediate (between $85 \%$ and $10 \%$ of time), peaking (less than $10 \%$ of time), and off units. The optimal maintenance schedule is shown in Fig. 7, in which much of the maintenance is scheduled near the minimum loading hours for maintaining the power systems reliability and economics. The maintenance of base units U10 and U20 is scheduled at the minimum loading hours of 481-504 and 457-480, respectively. Furthermore, the maintenance of line L51 is at hours 140-157, which shifts the maintenance of peaking unit U34 to the next available minimum loading hours of 289-312. The optimal cost is $\$ 40176827$ which includes the generator maintenance cost of $\$ 62400$, line maintenance cost of $\$ 90000$, and operation cost of $\$ 40024427$. Compared with the optimal cost of Case 1, a saving of $\$ 1956729$ is achieved due to the simultaneous consideration of LTEM and LTSCUC subproblems.

Case 3: In this case, the power system uncertainty is considered for the coordination of LTEM and LTSCUC problems.

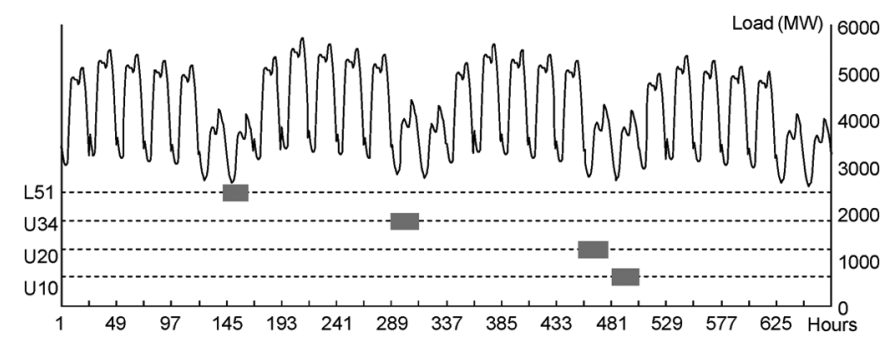

Fig. 7. Equipment maintenance in Case 2.

TABLE IV

Probabilities of EACH SCENARIO AFter SCENARIO REDUCTION

\begin{tabular}{l|c|c|c|c|c|c}
\hline Scenario & 1 & 2 & 3 & 4 & 5 & 6 \\
\hline Probability & 0.01 & 0.01 & 0.28 & 0.01 & 0.01 & 0.39 \\
\hline Scenario & 7 & 8 & 9 & 10 & 11 & 12 \\
\hline Probability & 0.01 & 0.01 & 0.01 & 0.01 & 0.01 & 0.24 \\
\hline
\end{tabular}

TABLE V

OPERATION COST FOR EACH SCENARIO (\$)

\begin{tabular}{c|c|c|c}
\hline Scenario & Operation Cost & Scenario & Operation Cost \\
\hline 1 & $40,767,940$ & 7 & $39,288,403$ \\
\hline 2 & $40,202,328$ & 8 & $41,929,654$ \\
\hline 3 & $40,984,806$ & 9 & $41,345,839$ \\
\hline 4 & $39,963,756$ & 10 & $41,724,055$ \\
\hline 5 & $40,717,038$ & 11 & $41,637,338$ \\
\hline 6 & $40,581,050$ & 12 & $41,283,492$ \\
\hline \multicolumn{2}{c}{ EXP } & \multicolumn{2}{c}{$0,806,157 \pm 64,223$} \\
\hline
\end{tabular}

At each hour, there are 461 decision variables and 2094 continuous variables. Thus the entire coordinated problem over a 672-h horizon contains 309792 binary variables and 1407168 continuous variables. One hundred scenarios are created using the low-discrepancy Monte Carlo simulation method with each scenario representing forced outages of generation units and transmission lines, load forecast errors, and fuel price fluctuations. The scenario reduction method reduces the total number of scenarios from 100 to 12 as a tradeoff between the calculation speed and the solution accuracy. Table IV shows the scenario weights after reduction. Table $\mathrm{V}$ provides the operation cost of each scenario, the expected operation cost, and its variance. In Table V and thereafter, "EXP" is the expected value of all scenarios based on the $95 \%$ confidence interval, and the "Relative Error" is calculated as the ratio of $95 \%$ confidence interval to the expected value. The results presented in Tables V show that after $90 \%$ reduction, relative errors in operation costs are less than $1 \%$.

Table VI compares maintenance cost, operation cost, and total cost for the above three cases. The coordination of LTEM and LTSCUC subproblems has reduced the total cost by $\$ 1956729$ in Case 2 as compared with Case 1. This reduction is due to the coordination of LTEM and LTSCUC subproblems which avoids any unnecessary load shedding or the commitment of peaking units. It also shows that the uncertainty has increased the total operation cost by $\$ 861730$ in Case 3 as compared with Case 2 . The inclusion of uncertainty would provide a more comprehensive coordination of maintenance outages and hourly unit commitment to satisfy the system security constraints. 
TABLE VI

COST COMPARISON IN THREE CASES

\begin{tabular}{l|c|c|c}
\hline Case & $\begin{array}{c}\text { Maintenance } \\
\text { Cost (\$) }\end{array}$ & $\begin{array}{c}\text { Operation } \\
\text { Cost (\$) }\end{array}$ & $\begin{array}{c}\text { Total } \\
\text { Cost (\$) }\end{array}$ \\
\hline Case 1 & 152,400 & $41,981,156$ & $42,133,556$ \\
\hline Case 2 & 152,400 & $40,024,427$ & $40,176,827$ \\
\hline Case 3 & 152,400 & $40,886,157$ & $41,038,557$ \\
\hline
\end{tabular}

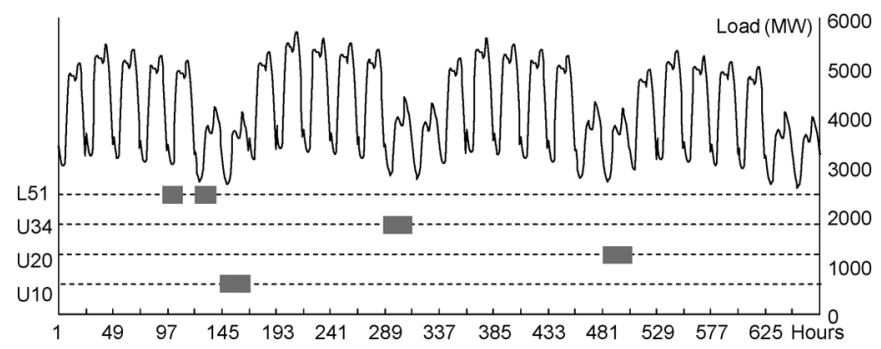

Fig. 8. Equipment maintenance in Case 3.

The optimal maintenance schedule is presented in Fig. 8. The load forecast in Fig. 8 is the same as that in Fig. 5. Among the simulated scenarios, the base unit U10 is on forced outage at hours 143-148 in scenario 6, and at hours 153-156 in scenario 10. Thus the optimal maintenance schedule of unit U10 is shifted to hours 145-168, and the maintenance of units U20 and U34 is shifted to hours 481-504 and 289-312, respectively.

Furthermore, the forced outage of transmission line L51 is simulated to be between hours $100-103$ in scenario 3 . Thus its maintenance outage is scheduled at periods of 97-105 and 126-134. The optimal cost is $\$ 41038557$ which includes the generator maintenance cost of $\$ 62400$, the transmission line maintenance cost of $\$ 90000$, and the operation cost of $\$ 40886157$.

To further evaluate the advantage of the stochastic model, a new set of scenarios is generated as shown in Table VII. We apply the stochastic problem solution (i.e., solution of Case 3) and the expected value problem solution (i.e., solution of Case 2) to each scenario. Tables VIII and IX show the operation costs for each scenario which are calculated by adopting the stochastic and the deterministic problem solutions of the maintenance and the unit commitment decision variables in each scenario. By comparison, the difference in the expected cost between stochastic and deterministic solutions is \$543397 (i.e., \$41 491 977-\$40 948 580), which shows the potential operation cost saving for utilizing the stochastic model. By comparison, the upper limit of the expected operation cost for the proposed stochastic solution (i.e., $\$ 40948580+\$ 78305=\$ 41026885$ ) is smaller than the lower limit of the expected operation cost of the deterministic decision (i.e., $\$ 41491977-\$ 105887=$ $\$ 41386090$ ), which exhibits a potential saving when applying the stochastic model. The scenario costs in Table VIII are not necessarily smaller than those in Table IX for the deterministic solution. The costs for scenarios 6, 9, and 11 in Table VIII are higher than those in Table IX.

Fig. 9 shows the relationship between maintenance and MCPs calculated by the LTSCUC subproblem in a four-week horizon. The maintenance is likely to be scheduled when MCPs are low.
TABLE VII

Probabilities of EACH SCENARIO AFTER SCENARIO REDUCTION

\begin{tabular}{l|c|c|c|c|c|c}
\hline Scenario & 1 & 2 & 3 & 4 & 5 & 6 \\
\hline Probability & 0.18 & 0.03 & 0.25 & 0.03 & 0.12 & 0.09 \\
\hline Scenario & 7 & 8 & 9 & 10 & 11 & 12 \\
\hline Probability & 0.01 & 0.03 & 0.04 & 0.04 & 0.02 & 0.16 \\
\hline
\end{tabular}

TABLE VIII

Operation Costs Using StOchastic VALUe SOlution (\$)

\begin{tabular}{c|c|c|c}
\hline Scenario & Operation Cost & Scenario & Operation Cost \\
\hline 1 & $40,713,112$ & 7 & $39,684,448$ \\
\hline 2 & $40,085,069$ & 8 & $41,868,003$ \\
\hline 3 & $40,774,148$ & 9 & $41,856,600$ \\
\hline 4 & $40,003,360$ & 10 & $41,450,497$ \\
\hline 5 & $40,660,151$ & 11 & $42,002,161$ \\
\hline 6 & $41,311,224$ & 12 & $41,259,936$ \\
\hline \multicolumn{2}{|c|}{ EXP } & \multicolumn{2}{c}{0.191} \\
\hline
\end{tabular}

TABLE IX

Operation Costs Using EXPECTEd VAlue SOLUtion (\$)

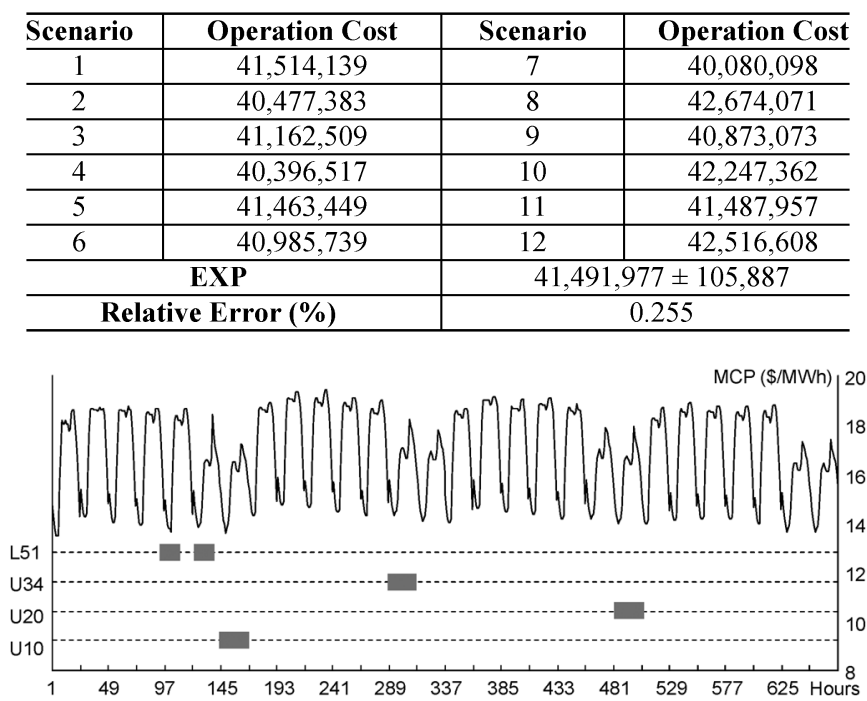

Fig. 9. Equipment maintenance in the stochastic case combined with MCP.

When units are on maintenance, expensive units have to be committed to meet system loads, which will increase certain hourly MCPs. The maintenance carried out at the low MCP periods could mitigate price risks in spot markets.

Figs. 10 and 11 show the iterative LR function values and the number of infeasible relaxed constraints (11) and (12), respectively. Fig. 11 shows that after 58 iterations of LTEM and LTSCUC, there are three violated coupling constraints corresponding to (11) and (12). We fix the maintenance schedule at iteration 58 and execute the LTSCUC to obtain a feasible and near-optimal solution of $\$ 41038557$. Fig. 10 shows that at iteration 58, the relative difference between $V L R(\$ 40865834)$ and $V P(\$ 41038557)$ is $0.423 \%$ which satisfies the precision requirement. For the stochastic LTSCUC, we show the convergence of the last stochastic LTSCUC subproblem.

Figs. 12 and 13 show the iterative LR function values of (17) and the weighted violation of bundle constraints, respectively. Fig. 13 shows that after 27 iterations, the total 


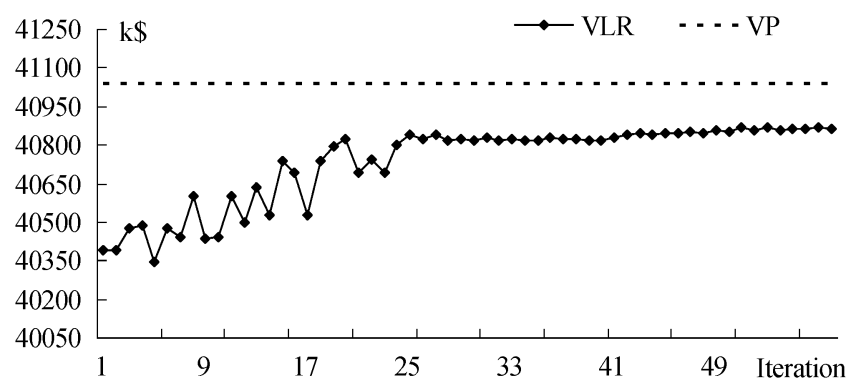

Fig. 10. Iterative LR function values in Case 3 .

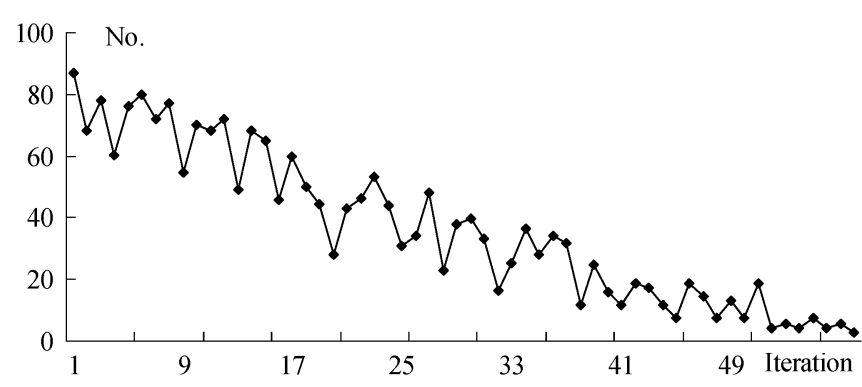

Fig. 11. Number of infeasible constraints (11), (12) in Case 3.

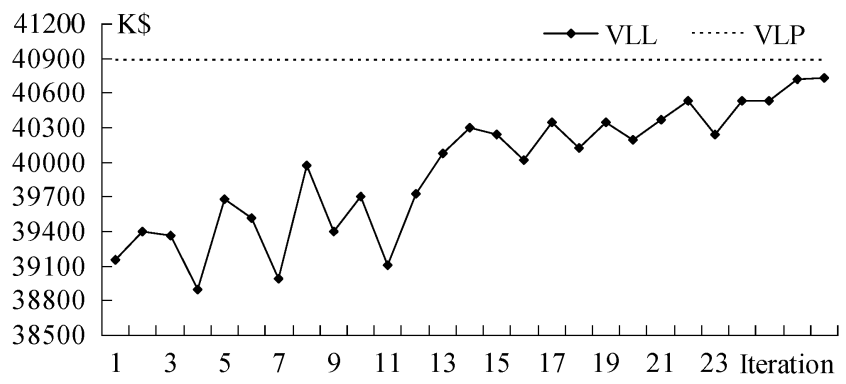

Fig. 12. Iterative LR values of (16) for one stochastic LTSCUC in Case 3.

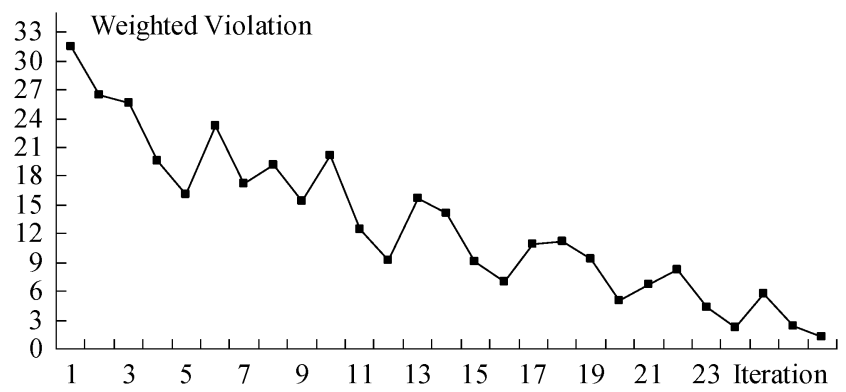

Fig. 13. Iterative weighted violation for one stochastic LTSCUC in Case 3.

weighted violation of bundle constraints is satisfied with a $V L L$ of $\$ 40731316$. Then, we apply the heuristic process to find a feasible solution with respect to the scenario bundle constraints. This process would require another 15 iterations and the relative difference between $V L L(\$ 40731316)$ and $V L P(\$ 40886157)$ is $0.380 \%$ as shown in Fig. 12.

Case 4: In this case, a three-day maintenance duration is studied. Other assumptions are the same as those of Cases $1-3$. Also, the power system uncertainty is modeled in this case. First, we consider the uncoordinated solution for LTEM and LTSCUC. The optimal maintenance schedule is shown in Fig. 14. The total cost is $\$ 42951648$ which includes the

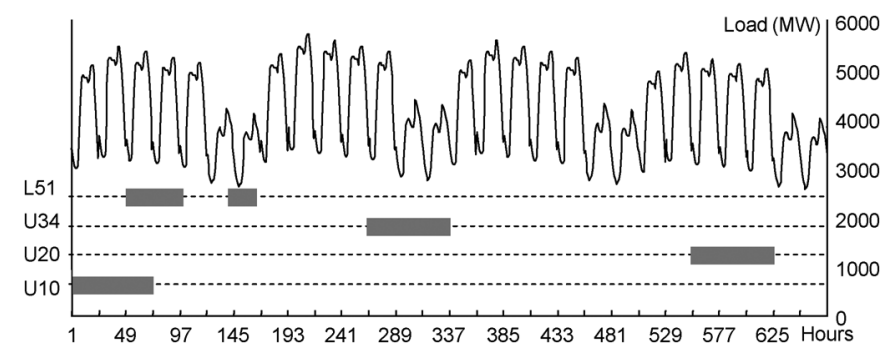

Fig. 14. Maintenance with uncoordinated LTEM and LTSCUC.

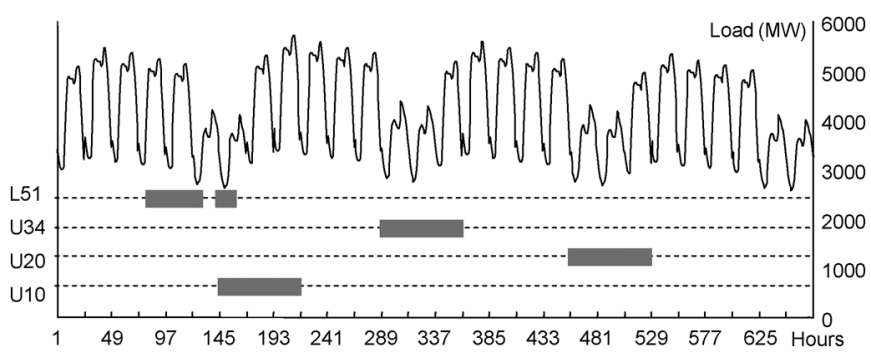

Fig. 15. Maintenance with coordinated LTEM and LTSCUC in Case 4.

generator maintenance cost of $\$ 187200$, the line maintenance cost of $\$ 360000$, and the operation cost of $\$ 42404448$. Note that the uncoordinated LTEM and LTSCUC solutions will result in the scheduling of maintenance at periods with higher loads and more expensive operation costs. Next we consider the coordination of LTEM and LTSCUC solutions. The optimal maintenance schedule is shown in Fig. 15. Thus the optimal maintenance of unit U10 is scheduled at hours 145-216, which do not coincide with the low-load period at hours 121-144, and maintenance of units U20 and U34 is scheduled at hours $457-528$ and $288-360$, respectively. The total cost is $\$ 41798521$ which includes the generator maintenance cost of $\$ 187200$, the line maintenance cost of $\$ 360000$, and the operation cost of $\$ 41251321$.

As we compare the coordinated and the uncoordinated results, a saving of $\$ 1153127$ (i.e., 42951648 - 41798521 ) occurs due to the coordination of LTEM and LTSCUC. Furthermore, the inclusion of uncertainty provides a more accurate coordination of maintenance outage and hourly generation unit commitment, satisfies the system security constraints, and makes a more efficient usage of fuel contracts.

The cases are tested on a 2.39-GHz server and the execution time is estimated based on parallel calculations with 12 CPUs. The CPU time of LTSCUC for one scenario is about 4 min, and the number of iterations for scenario bundle constraints is within 27 for the given accuracy. The heuristic process for obtaining a feasible LTSCUC solution requires another 15 iterations and $15 * 0.2$ minutes of computation. Thus the total CPU time for one iteration of LTEM and LTSCUC is about $1.9 \mathrm{~h}(4 * 27+15 * 0.2 \mathrm{~min})$, with the parallel processing of LTEM and stochastic LTSCUC subproblems. Notice that only the objective functions are changed in the following iterations, and the solutions obtained at previous iterations are still feasible which can be used as initial solutions in the current iteration. Furthermore, previous Benders cuts can also be used at the current LTSCUC calculation. Such strategies would reduce 
the computation time. However, these strategies would not be suitable for the last iteration, which is a heuristic process for obtaining the feasibility solution by fixing the LTEM solutions and executing the LTSCUC. In Case 3, the total number of iterations between LTEM and LTSCUC is 58, and the total CPU time is about $57 \mathrm{~h}$. However, if parallel computation is further adopted in each short-term SCUC calculation process, the total CPU time would be reduced to about 4.6 hours. In general, the computation time may not be a major concern since the proposed model is applied to the long-term evaluation. For a complex stochastic mixed-integer linear problem presented in the paper, the proposed decomposition methodology would provide a reliable solution with an acceptable duality gap within a reasonable CPU time.

\section{CONCLUSIONS}

Due to random events in power systems, a stochastic problem is proposed in this paper which coordinates LTEM and LTSCUC solutions with fuel and emission constraints. The approach considers the uncertainty in the availability of generation units and transmission lines, load forecast errors, as well as fuel price fluctuations, which are simulated as scenario trees by the Monte Carlo Method. LR is applied to decompose the original problem into maintenance and unit commitment subproblems, multipliers $\eta$ and $\pi$ corresponding to generator and line status coupling constraints are updated and fed back to the maintenance and unit commitment subproblems. The uncertainty is represented in the unit commitment scenarios, and LR is applied to solve the stochastic unit commitment problem with fuel allocation and emission allowance constraints. The solution results for the hourly maintenance and unit commitment subproblems represent the coordination of security, optimal unit commitment, resource allocation, and equipment maintenance in uncertain power systems. Compared with the expected value problem solution, the solution of the proposed stochastic model would provide potential savings in operation costs. The proposed approach can be applied to a vertically integrated utility as well as the ISO's operation in a restructured power system.

\section{APPENDIX A}

The process starts by finding a scenario $s$ which has the largest weighted violation $\sum_{p=1}^{N P} \sum_{t=1}^{N T} P^{s} \cdot\left|I_{i t p}^{s}-c_{i t p}\right|$, updating multipliers, and calculating the scenario $s$ only. The steps are as follows:

Step 0: Calculate $I_{i t p}^{s}$ for each scenario with $s=$ $1, \cdots, S$. Compute $c_{i t p}=\sum_{s \in B(s, P, \tau)=\Omega_{P \tau}} P^{s}$. $I_{i t p}^{s} / \sum_{s \in B(s, P, \tau)=\Omega_{P \tau}} P^{s}$.

Step 1: Update multipliers. The multipliers are updated as $\theta_{i t p}^{s, k+1}=\theta_{i t p}^{s, k}+r^{k} \cdot d_{i t p}^{s, k}$, where $r^{k}$ is the step size which satisfies $0 \leq r^{k} \leq\left(L^{*}-L^{k}\right) /\left\|d^{k}\right\|^{2}$ and $d_{i t p}^{s, k}$ is the modified surrogate subgradient, which is given by

$$
\begin{aligned}
& d_{i t p}^{s, 0}=I_{i t p}^{s, 0}-c_{i t p}^{0} \\
& d_{i t p}^{s, k}=\left[I_{i t p}^{s, k}-c_{i t p}^{k}\right]+\gamma_{i t p}^{s, k} \cdot\left[I_{i t p}^{s, k-1}-c_{i t p}^{k-1}\right]
\end{aligned}
$$

where $\gamma_{i t p}^{s, k}=\max \left\{0,-\varsigma \cdot\left(I_{i t p}^{s, k}-c_{i t p}^{k}\right) / d_{i t p}^{s, k-1}\right\} \varsigma \in[1,2]$.
Step 2: Use new multipliers to recalculate the problem, get new solutions for $I_{i t p}^{s}$.

Step 3: Check the stopping criteria. If the criterion given by $\sum_{s \in B(s, P, \tau)=\Omega_{P \tau}} P^{s} \sum_{p=1}^{N P} \sum_{t=1}^{N T}\left|I_{i t p}^{s}-c_{i t p}\right| \leq \varepsilon_{2}$ is met, then stop; otherwise, go to Step 1.

\section{APPENDIX B}

Proof for Stopping Criterion: The original optimization problem is defined as

$$
\begin{aligned}
& \text { VP } P^{*}=\operatorname{Min}\left\{f(x)+\sum_{s} g\left(y^{s}\right)\right\} \\
& \text { S.t. } \quad x \in X \quad y^{s} \in Y^{s} \\
& y^{s} \leq x \quad \forall s \\
& y^{s}=y^{s^{\prime}} \quad \text { for } \quad s \in B(s, P, \tau)=\Omega_{P \tau} .
\end{aligned}
$$

The Lagrangian dual of the primal problem (B1) is written as

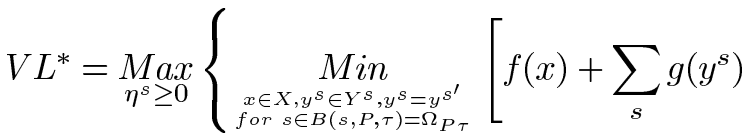

$$
\begin{aligned}
& \left.\left.+\sum_{s} \eta^{s} \cdot\left(y^{s}-x\right)\right]\right\} \text {. }
\end{aligned}
$$

We minimize the following Lagrangian function (B3) at the current iteration $n$ which is a lower bound of (B2)

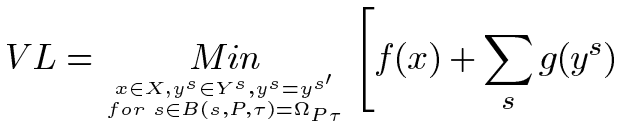

$$
\begin{aligned}
& \left.+\sum_{s} \eta^{s, n} \cdot\left(y^{s}-x\right)\right]
\end{aligned}
$$

Let $V P$ be the objective value of a feasible solution of the original problem (B1), which is the upper bound of $V P^{*}$. Based on the above discussions

$$
V L \leq V L^{*} \leq V P^{*} \leq V P
$$

(B3) is further decomposed into two subproblems as (B5) and (B6) corresponding to $x$ and $y$, respectively:

$$
\begin{aligned}
V L_{x} & =\operatorname{Min}_{x \in X}\left[f(x)-\sum_{s}\left(\eta^{s, n} \cdot x\right)\right] \\
V L_{y} & =\operatorname{Min}\left[\sum_{s} g\left(y^{s}\right)+\sum_{s}\left(\eta^{s, n} \cdot y^{s}\right)\right] \\
\text { S.t. } \quad y^{s} & \in Y^{s} \\
y^{s} & =y^{s^{\prime}} \quad \text { for } \quad s \in B(s, P, \tau)=\Omega_{P \tau} .
\end{aligned}
$$

Based on the discussion in Section IV-C, the Lagrangian dual of (B6) is

$$
\begin{aligned}
V L L_{y}^{*}=\operatorname{Max}_{\theta^{s}}\left\{\operatorname{Min}_{y^{s} \in Y^{s}}\right. & {\left[\sum_{s} g\left(y^{s}\right)+\sum_{s}\left(\eta^{s, n} \cdot y^{s}\right)\right.} \\
+\sum_{s \in B(s, P, \tau)}=\Omega_{P \tau} & \left.\left.\left(\theta^{s} \cdot y^{s}\right)\right]\right\} .
\end{aligned}
$$


In this paper, we minimize the following Lagrangian function at the current iteration $k$ which is the lower bound of (B7):

$$
\begin{aligned}
V L L_{y}=\operatorname{Min}_{y^{s} \in Y^{s}}\left[\sum_{s} g\left(y^{s}\right)\right. & +\sum_{s} \eta^{s, n} \cdot y^{s} \\
& \left.+\sum_{s \in B(s, P, \tau)=\Omega_{P \tau}}\left(\theta^{s, k} \cdot y^{s}\right)\right] .
\end{aligned}
$$

Accordingly

$$
V L L_{y} \leq V L L_{y}^{*} \leq V L_{y} .
$$

Combining (B4)-(B6) and (B9), we get

$$
\begin{array}{r}
V L L_{y}+V L_{x} \leq V L L_{y}^{*}+V L_{x} \leq V L_{y}+V L_{x} \\
=V L \leq V L^{*} \leq V P^{*} \leq V P .
\end{array}
$$

Thus the stopping criterion (B11) is used to terminate the iterative process since the unknown optimal solution $V P^{*}$ is between $\left(V L L_{y}+V L_{x}\right)$ and $V P$ :

$$
\frac{\left|V P-\left(V L L_{y}+V L_{x}\right)\right|}{\left(V L L_{y}+V L_{x}\right)} \leq \varepsilon_{1} \text {. }
$$

\section{REFERENCES}

[1] M. Shahidehpour and Y. Wang, Communication and Control of Electric Power Systems. New York: Wiley, Jun. 2003.

[2] M. Shahidehpour, H. Yamin, and Z. Y. Li, Market Operations in Electric Power Systems. New York: Wiley, 2002.

[3] M. Shahidehpour and M. Marwali, Maintenance Scheduling in Restructured Power Systems. Norwell, MA: Kluwer, 2000.

[4] E. L. Silva, M. Morozowski, L. G. S. Fonseca, G. C. Oliveira, A. C. G. Melo, and J. C. O. Mello, "Transmission constrained maintenance scheduling of generating units: A stochastic programming approach," IEEE Trans. Power Syst., vol. 10, no. 2, pp. 695-701, May 1995.

[5] R. Billinton and A. Abdulwahab, "Short-term generating unit maintenance scheduling in a restructured power system using a probabilistic approach," Proc. Inst. Elect. Eng., Gen., Transm., Distrib., vol. 150, no. 4, pp. 463-468, Jul. 2003.

[6] A. J. Conejo, R. Garcia-Bertrand, and M. Diaz-Salazar, "Generation maintenance scheduling in restructured power systems," IEEE Trans. Power Syst., vol. 20, no. 2, pp. 984-992, May 2005.

[7] T. Geetha and K. S. Swarup, "Coordinated maintenance scheduling of Gencos and Transcos in restructured power systems," in Proc. IEEE Power India Conf., 2006.

[8] M. K. C. Marwali and M. Shahidehpour, "Coordination between longterm and short-term generation scheduling with network constraints," IEEE Trans. Power Syst., vol. 15, no. 3, pp. 1161-1167, Aug. 2000.

[9] Y. Fu, M. Shahidehpour, and Z. Li, "Long-term security-constrained unit commitment: Hybrid subgradient and Danzig-Wolfe decomposition," IEEE Trans. Power Syst., vol. 20, no. 4, pp. 2093-2106, Nov. 2005.

[10] J. Lenzuela and M. Mazumdar, "Monte Carlo computation of power generation production costs under operating constraints," IEEE Trans. Power Syst., vol. 16, no. 4, pp. 671-677, Nov. 2001.

[11] P. Glasserman, Monte Carlo Method in Financial Engineering. New York: Springer, 2003.

[12] Y. Yamamoto and T. Tezuka, "Optimal investment in power plant under price uncertainty," in Proc. 41st SICE Annu. Conf. (SICE 2002), Aug. 2002, vol. 2, pp. 1302-1305.
[13] J. Dupăová, N. Gröwe-Kuska, and W. Römisch, "Scenario reduction in stochastic programming: An approach using probability metrics," Math. Program., ser. A, vol. 95, pp. 493-511, 2003.

[14] R. T. Rockafellar and R. J.-B. Wets, "Scenarios and policy aggregation in optimization under uncertainty," Math. Oper. Res., vol. 16, no. 1, pp. 119-147, 1991

[15] X. Zhao, P. B. Luh, and J. Wang, "Surrogate gradient algorithm for lagrangian relaxation," J. Optim. Theory Appl., vol. 100, no. 3, pp. 699-712, Mar. 1999.

[16] P. Camerini, L. Fratta, and F. Maffioli, "On improving relaxation methods by modified gradient techniques," in Mathematical Programming Study. Amsterdam, The Netherlands: North Holland, 1975, vol. 3, pp. 26-34.

[17] M. Shahidehpour, Y. Fu, and T. Wiedman, "Impact of natural gas infrastructure on electric power systems," Proc. IEEE, vol. 93, no. 5, pp. 1042-1056, May 2005.

[18] Y. Fu, M. Shahidehpour, and Z. Li, "Security-constrained unit commitment with AC constraints," IEEE Trans. Power Syst., vol. 20, no. 3 , pp. 1538-1550, Aug. 2005

[19] D. P. Bertsekas, Nonlinear Programming. Belmont, CA: Athena Scientific, 1999

[20] L. Wu, M. Shahidehpour, and T. Li, "Stochastic security-constrained unit commitment," IEEE Trans. Power Syst., vol. 22, no. 2, pp. 800-811, May 2007.

[21] L. Wu, M. Shahidehpour, and Z. Li, "GENCO's risk-constrained hydrothermal scheduling," IEEE Trans. Power Syst., vol. 23, no. 4, pp. 1847-1858, Nov. 2008.

[22] L. Wu, M. Shahidehpour, and T. Li, "Cost of reliability analysis bases on stochastic unit commitment," IEEE Trans. Power Syst., vol. 23, no. 3, pp. 1364-1374, Aug. 2008.

[23] Y. Fu, Z. Li, M. Shahidehpour, T. Zheng, and E. Litvinov, "Coordination of midterm outage scheduling with short-term security-constrained unit commitment," IEEE Trans. Power Syst., vol. 24, no. 4 , pp. 1818-1830, Nov. 2009.

[24] J. R. Birge and F. V. Louveaux, Introduction to Stochastic Programming. New York: Springer, 1997.

Lei Wu (M'07) received the B.S. and M.S. degrees in electrical engineering from Xi' an Jiaotong University, Xi'an, China, in 2001 and 2004, respectively, and the $\mathrm{Ph} . \mathrm{D}$. degree in electrical engineering from the Illinois Institute of Technology, Chicago, in 2008 .

Presently, he is a Visiting Professor at Illinois Institute of Technology. His research interests include power systems restructuring, reliability, and economics.

Mohammad Shahidehpour (F'01) is Bodine Chair Professor in the Electrical and Computer Engineering Department at Illinois Institute of Technology, Chicago. He is an Honorary Professor in the North China Electric Power University in Beijing and the Sharif University in Tehran. He is also the 2009 recipient of an Honorary Doctorate from the Polytechnic University of Bucharest.

Dr. Shahidehpour is the VP of Publications for the IEEE Power \& Energy Society, an IEEE Distinguished Lecturer, Technical Program Chair for the 2010 IEEE Innovative Smart Grid Technologies Conference, and the Editor-in-Chief of the IEEE TRANSACTIONS ON SMART GRID.

Yong Fu (M'05) received the B.S. and M.S. degrees in electrical engineering from Shanghai Jiaotong University, Shanghai, China, in 1997 and 2002, respectively, and the Ph.D. degree in electrical engineering from the Illinois Institute of Technology, Chicago, in 2006.

Presently, he is an Assistant Professor in the Electrical and Computer Engineering Department at the Mississippi State University. His research interests include power systems restructuring and reliability. 